\title{
SIMULATIONS OF PARAMETRIC RESONANCE IONIZATION COOLING OF MUON BEAMS *
}

\author{
Kevin Beard", S. Alex Bogacz, Yaroslav Derbenev (Jefferson Lab, Newport News, Virginia), \\ Katsuya Yonehara (Illinois Institute of Technology, Chicago, Illinois), \\ Rolland P. Johnson, Kevin Paul, Thomas J. Roberts (Muons, Inc, Batavia)
}

\begin{abstract}
Parametric-resonance ionization cooling (PIC) is being developed to create small beams so that high muon collider luminosity can be achieved with fewer muons. In the linear channel that is studied in this effort, a half integer resonance is induced such that the normal elliptical motion of particles in $x-x^{\prime}$ phase space becomes hyperbolic, with particles moving to smaller $x$ and larger $x^{\prime}$ as they pass down the channel. Thin absorbers placed at the focal points of the channel then cool the angular divergence of the beam by the usual ionization cooling mechanism where each absorber is followed by RF cavities. Thus the phase space of the beam is compressed in transverse position by the dynamics of the resonance and its angular divergence is compressed by the ionization cooling mechanism. We report the first results of simulations of this process, a study of the compensation of chromatic aberration by using synchrotron oscillations.
\end{abstract}

\section{INTRODUCTION}

The concepts and basic equations for PIC are described in another paper submitted to this conference [1]. The essential practical question for PIC is how well detuning effects can be compensated. Chromatic aberration, the detuning effect we consider here, is where the momentum-dependent betatron frequency causes off- momentum particles to be out of resonance with the focusing lattice. Recent analytic studies [2] show that by choosing suitable synchrotron motion parameters, the resonance condition can be maintained. This paper reports the first simulation to test this prediction using the OptiM program [3].

\section{SOLENOID TRIPLET CELL}

Our studies of optimum lattice configurations indicate that an excellent cell for PIC consists of an alternating solenoid triplet. This provides strong focusing, so that the horizontal and vertical betatron phases advance by $3 \pi$ across the cell and by $\pi$ between the absorbers (indicated by markers). This configuration provides a periodic half-integer and integer resonant lattice.

The total length of the $\mathrm{p}=286.8 \mathrm{MeV} / \mathrm{c}$ cell is $7.2 \mathrm{~m}$ with the Initial betas of $21 \mathrm{~cm}$. In the transfer matrix

* Supported in part by DOE SBIR grants DE-FG02-03ER83722, and 04ER84016.

\# Beard@JLab.org formalism of OptiM the three "soft edge" solenoids have fields of $\mathrm{B}_{0}=-34.1,32.4$, and $-34.1 \mathrm{kG}$ with lengths $\mathrm{L}=80,130$, and $80 \mathrm{~cm}$, respectively, and radius $\mathrm{a}=20 \mathrm{~cm}$. The Larmor wave number is $\mathrm{k}=\mathrm{e} \mathrm{B}_{0} / \mathrm{Pc}$.

The periodic solution of the cell is illustrated in terms of Twiss functions below:
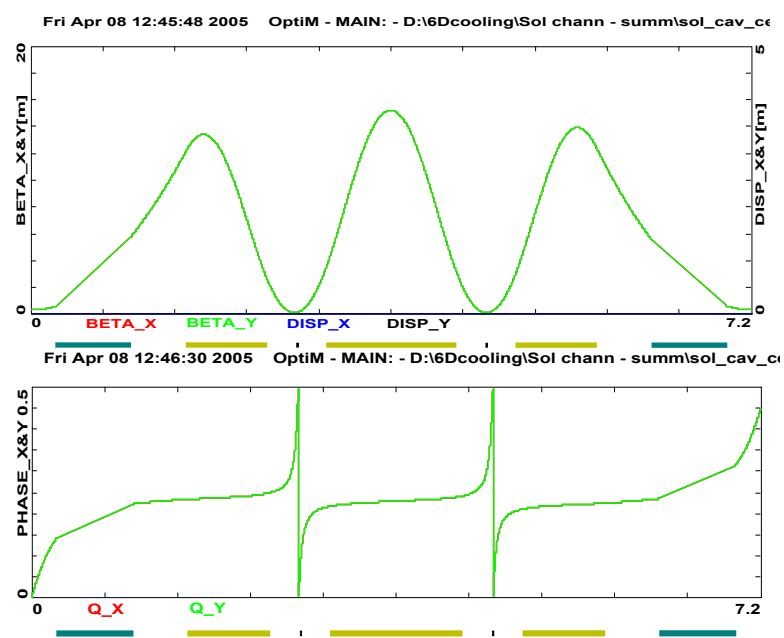

Figure 1: Beta functions and phases for the solenoid triplet cell. Thin absorbers are placed at the two central focal points. In the simulations for Fig. 2 the lost energy is simply replaced at the absorbers. For Fig. 3, $400 \mathrm{MHz}$ RF cavities shown as blue bars replace the lost energy and provide synchrotron motion.

\section{'Soft edge” Solenoid-matrix implementation}

If the aperture is equal to zero a solenoid is considered as an ideal linear solenoid with transfer matrix equal to

$$
\mathbf{M}_{\mathrm{sol}}=\left[\begin{array}{cccc}
\frac{1+\cos (k L)}{2} & \frac{\sin (k L)}{k} & \frac{\sin (k L)}{2} & \frac{1-\cos (k L)}{k} \\
-\frac{k \sin (k L)}{4} & \frac{1+\cos (k L)}{2} & -k \frac{1-\cos (k L)}{4} & \frac{\sin (k L)}{2} \\
-\frac{\sin (k L)}{2} & -\frac{1-\cos (k L)}{k} & \frac{1+\cos (k L)}{2} & \frac{\sin (k L)}{k} \\
k \frac{1-\cos (k L)}{4} & -\frac{\sin (k L)}{2} & -\frac{k \sin (k L)}{4} & \frac{1+\cos (k L)}{2}
\end{array}\right] .
$$

If the aperture is non-zero there is a correction related to the finite length of the solenoid edge. This correction decreases the solenoid total focusing in comparison with the ideal solenoid of the same length. In this case the solenoid length is determined by:

$$
L=\frac{1}{B_{0}} \int_{-\infty}^{\infty} B_{z}(s) d s \text {. }
$$


The correction to the edge focusing at each solenoid edge is determined by the following formula:

$$
\Phi_{\text {edge }}=\frac{1}{2}\left(\int_{-\infty}^{\infty} B_{z}^{2}(s) d s-B_{0}^{2} L\right)=-\frac{k^{2} a}{8} .
$$

Therefore, the edge focusing of a solenoid can be described by an axially symmetric multipole $(\mathrm{m}=-1)$ through the following transfer matrix:

$$
\begin{gathered}
\mathbf{M}_{\text {edge }}=\left[\begin{array}{cccc}
1 & 0 & 0 & 0 \\
-\Phi_{\text {edge }} & 1 & 0 & 0 \\
0 & 0 & 1 & 0 \\
0 & 0 & -\Phi_{\text {edge }} & 1
\end{array}\right], \\
\Phi_{\text {edge }}=-\frac{k^{2} a}{8}
\end{gathered}
$$

The final transfer matrix describing a 'soft edge' solenoid can be expressed as:

$$
\mathbf{M}_{\text {soft sol }}=\mathbf{M}_{\text {edge }} \mathbf{M}_{\text {sol }} \mathbf{M}_{\text {edge }} \text {. }
$$

\section{THIN ABSORBER AND RF}

Ionization cooling due to energy loss $(-\Delta p)$ in a thin absorber followed by immediate re-acceleration $(\Delta \mathrm{p})$ can be described as:

$$
\Delta \theta_{\perp}=-\theta_{\perp} \frac{\Delta p}{p}
$$

The corresponding canonical transfer matrix can be written as

$$
\begin{gathered}
M_{a b s}=K\left[\begin{array}{cccc}
1 & 0 & 0 & 0 \\
0 & 1-\frac{\Delta p}{p} & 0 & 0 \\
0 & 0 & 1 & 0 \\
0 & 0 & 0 & 1-\frac{\Delta p}{p}
\end{array}\right] K^{-1} \\
K=\left[\begin{array}{cccc}
1 & 0 & 0 & 0 \\
0 & 1 & -k / 2 & 0 \\
0 & 0 & 1 & 0 \\
k / 2 & 0 & 0 & 1
\end{array}\right] \quad \hat{\mathbf{x}} \equiv\left[\begin{array}{c}
x \\
p_{x} \\
y \\
p_{y}
\end{array}\right] \quad \mathbf{x} \equiv\left[\begin{array}{c}
x \\
\theta_{x} \\
y \\
\theta_{y}
\end{array}\right]
\end{gathered}
$$

where $k=e B_{z} / p c$ and $\hat{\mathbf{x}}=K \mathbf{x}$.

\begin{tabular}{|c|l|l|}
\hline normalized emittance: $\varepsilon_{\mathrm{x}} / \varepsilon_{\mathrm{y}}$ & $\mathrm{mm}$ & 30 \\
\hline $\begin{array}{c}\text { longitudinal emittance: } \varepsilon_{1} \\
\left(\varepsilon_{1}=\sigma_{\Delta \mathrm{p}} \sigma_{\mathrm{z}} / \mathrm{m}_{\mu} \mathrm{c}\right)\end{array}$ & $\mathrm{mm}$ & 0.8 \\
\hline momentum spread: $\sigma_{\Delta \mathrm{p} / \mathrm{p}}$ & & 0.01 \\
\hline bunch length: $\sigma_{\mathrm{z}}$ & $\mathrm{mm}$ & 30 \\
\hline momentum & $\mathrm{MeV} / \mathrm{c}$ & 286.8 \\
\hline
\end{tabular}

Table 1: Initial parameters for simulation studies.

In the simulation $\Delta p / p$ of 0.05 was used to mock-up the effect of a thin $(4 \mathrm{~cm})$ Be plate followed by reacceleration $(\Delta \mathrm{p}=14 \mathrm{MeV} / \mathrm{c})$.
To induce synchrotron oscillation into the channel dynamics two $400 \mathrm{MHz}$ RF cavities at zero crossing are added symmetrically to each cell. The cavity gradient of $17.3 \mathrm{MeV} / \mathrm{m}$ was chosen to provide appropriate height of a stationary bucket with a synchrotron phase advance of about $2 \pi / 8$ per cell. Since the RF cavities in the simulation provide some focusing, it was necessary to reset the triplet solenoid values to reestablish the resonant condition $(\mathrm{B}=-33.9$, $32.1,-33.9)$

\section{SIMULATIONS}

The simulation involves tracking 5000 particles defined by a $6 \mathrm{D}$ Gaussian with the parameters from the Table through a PIC channel consisting of 8 periodic cells (two absorbers per cell), or one synchrotron oscillation period. A sequence of transverse $\left(x, x^{\prime}\right)$ and longitudinal $(\mathrm{s}, \mathrm{dp} / \mathrm{p} \times 1000)$ phase space 'snapshots' starting with the initial distribution and followed by the 'snapshots' taken after passing through two cells is collected is shown in Figs 2 and 4. In Fig. 2 the beam energy lost in the absorber is simply replaced as described in the previous section. In the simulations shown in Fig. 4, RF cavities are used to generate synchrotron motion as well as to replace the energy lost in the absorber.

\section{CONCLUSIONS}

Comparing Fig. 3 with Fig. 2 of the betatron and synchrotron phase space evolution through 8 solenoid triplet cells, it is easy to see that the synchrotron motion makes a significant difference. In Fig. 2 the tails of the betatron distribution are larger and particles are lost. In Figs 3 and 4 , the final $x-x^{\prime}$ plot shows the promise of PIC: the $x$ distribution has narrowed from the initial one, and the $x^{\prime}$ distribution shows the effects of ionization cooling. However, the evaluation of the actual cooling of the beam awaits a proper simulation using all the power of the most realistic codes.

The simulation model used here has provided a first confirmation that our understanding of PIC is correct and that compensation for chromatic aberrations can be made as suggested. We will soon test the theory that angular aberrations can be compensated as well and include multiple scattering and energy straggling effects using G4Beamline [4].

\section{REFERENCES}

[1] Yaroslav Derbenev and Rolland P. Johnson, Ionization Cooling Using a Parametric Resonance, this conference.

[2]Y. Derbenev and R. P. Johnson, Phys. Rev. ST Accel. Beams 8, 041002 (2005).

[3] V. Lebedev, http://www-

bdnew.fnal.gov/pbar/organizationalchart/lebedev/OptiM/optim.htm [4] T.Roberts, http://www.muonsinc.com/g4beamline.html 

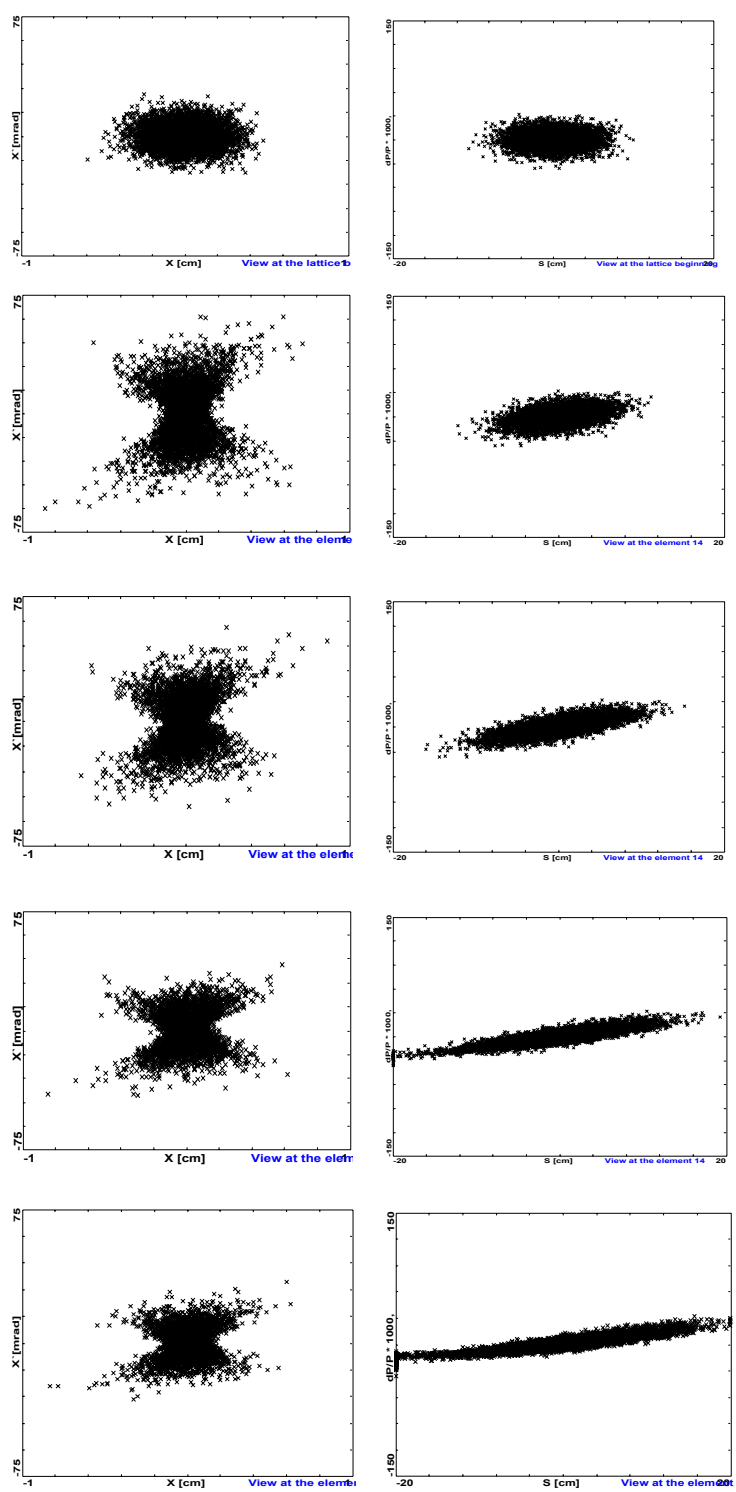

Figure 2: PIC simulation "snapshots" of $x-x^{\prime}$ (LEFT) and $s-\Delta p / p$ (RIGHT) phase space without synchrotron motion to correct chromatic aberration. Reading from top to bottom, each snapshot corresponds to passage through two of the cells shown in Fig. 1.

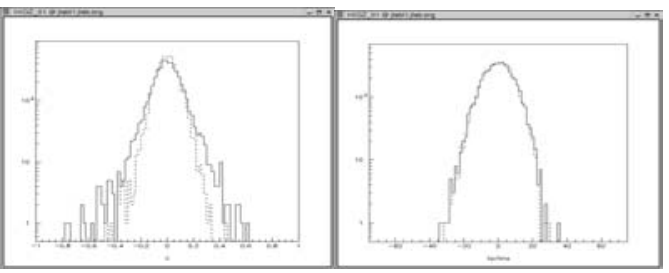

Figure 3: The left histogram displays the final $x$ distribution in $\mathrm{cm}$ with a solid line for the case without, and a dotted line for the case with, synchrotron RF. The right histogram displays the $x$ distribution in milliradians for the two cases.
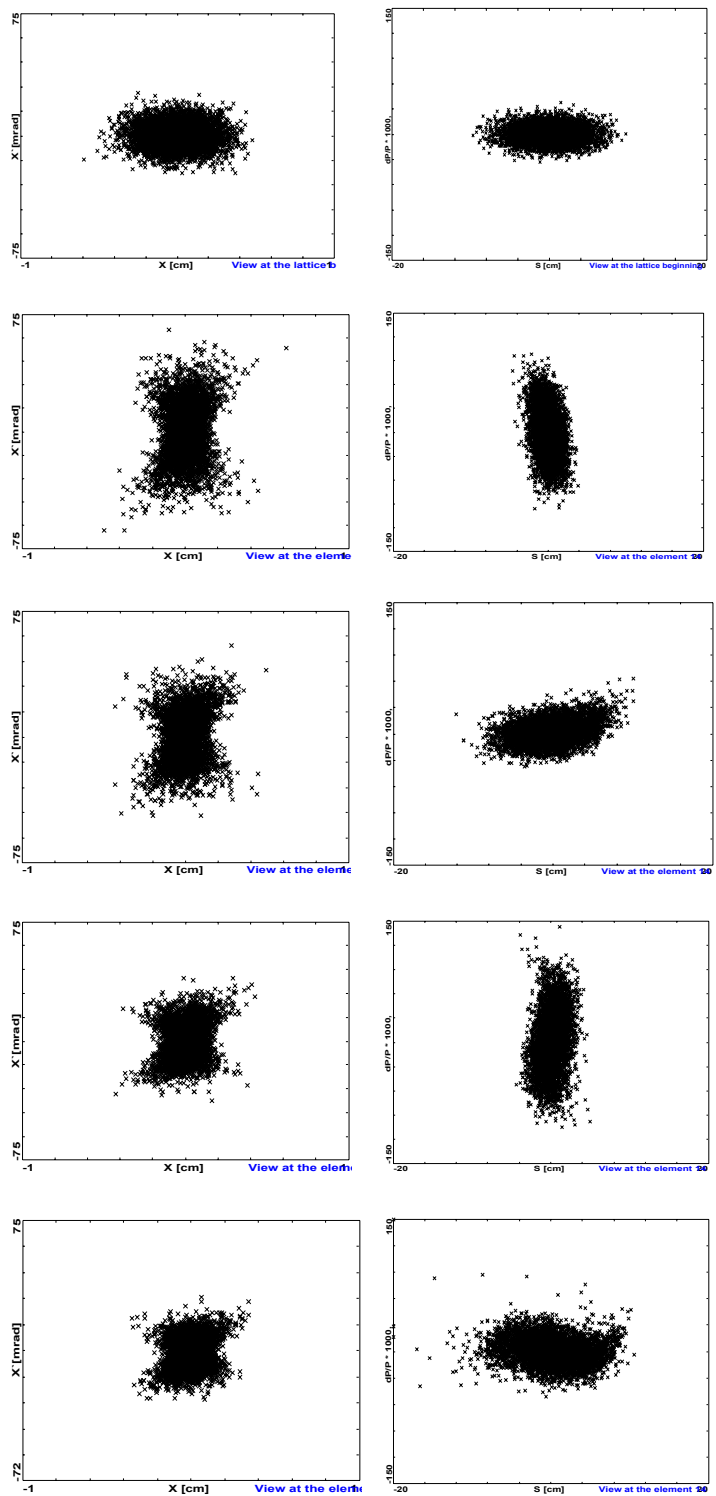

Figure 4: PIC simulation "snapshots" of $x-x^{\prime}$ (LEFT) and $s-\Delta p / p$ (RIGHT) phase space with synchrotron motion compensation of chromatic aberration. The transverse phase space with synchrotron motion is seen to be smaller than in Fig. 2. The RF bunch rotation of one synchrotron period is seen on the RIGHT plots. The growth of the longitudinal emittance is consistent with the conclusion that emittance exchange and longitudinal cooling will have to be combined with PIC. 